\title{
Heterogeneous Network Polymers. XI. Poly(L-glutamic acid) Crosslinked with Polyether Diisocyanates
}

\author{
Takehide TANAKA, Tetsuo Mori, Katsumi OGAWA, \\ and Ryuichi TANAKA \\ Department of Materials Science and Technology, Kyushu University, \\ Fukuoka 812, Japan.
}

(Received May 4, 1979)

\begin{abstract}
A series of heterogeneous network polymers [PGA-PEGDI] were synthesized by crosslinking poly(L-glutamic acid) [PGA] with hexamethylenediisocyanate-terminated poly(oxyethylene glycol) [PEGDI] in dimethylacetamide at $70^{\circ} \mathrm{C}$, while the solvent was allowed to evaporate slowly. The crosslinking reaction completed before the polymer concentration reached 20 percent. Since this concentration was lower than that of the ' $A$ ' point, the lyotropic cholesteric liquid-crystal structures involving PGA, which were prevalent in another series of heterogeneous network polymers [PGA-PEG] previously prepared from PGA and poly(oxyethylene glycol) [PEG], were prevented from forming in the PGA-PEGDI casting solutions. Thus, when brought to solid films, PGA-PEGDI was much less ordered than the PGA-PEG in which the 'solidified' liquid crystals persisted, as was evidenced by polarizing optical micrographs and X-ray diffraction patterns. The dynamic mechanical as well as the stress-strain behavior of PGA-PEGDI was also consistent with the idea that efficient admixture of the two component polymers could be accomplished in the stresscarrying continuous phase as a result of early crosslinking in the casting solutions.

KEY WORDS Heterogeneous Networks / Crosslinking / Poly(glutamic acid) / Polyether Diisocyanates / Liquid Crystal / Mechanical Properties /
\end{abstract}

Crosslinked polymers whose three-dimensional networks comprise two or more distinct kinds of polymeric chains often give rise to novel mechanical properties and are beginning to find industrial applications. The interpenetrating polymer networks $^{1-4}$ and Bamford's $\mathrm{ABCPs}^{5}$ are among the most extensively studied. Our heterogeneous network polymers are of the latter type, the component polymers being the hard, rod-like poly(L-glutamic acid) (PGA) and the flexible poly(oxyethylene glycol) (PEG).

Previously, crosslinking was brought about by esterifying $\gamma$-carboxyl groups of PGA with hydroxyl groups of PEG. $^{6}$ such polymers are called PGA-PEG heterogeneous network polymers. Their mechanical properties are essentially governed by the relative amounts of PGA and PEG; various individual factors such as compatibility, phase structure, crosslink density, residual free-carboxyl-group content, molecular weight of PEG, etc. are mutually dependent and collectively influence the mechanical behavior. ${ }^{7-10}$ Since the slow, dehydrative crosslink- ing reaction does not occur until almost all the solvent evaporates, the essential features of the phases in the crosslinked solid films remain prectically the same as those observed in the almost dry, but noncrosslinked films. ${ }^{11.12}$ Namely, the cholesteric liquid-crystal structures and the phase separation, which develop as the solvent evaporates, are retained in the crosslinked films to varying extents, depending delicately on the composition of the original casting mixtures, the rate of solvent evaporation, etc. $^{12}$

Recently, we discovered that polyether diisocyanates, which are often used to manufacture polyurethanes, can readily crosslink PGA via the decarboxylative amide forming reaction between $\mathrm{COOH}$ and $\mathrm{NCO}$ groups under much milder conditions than those for the preparation of PGA-PEG. The crosslinking proceeds even in dilute solutions and its completion appears to precede any appreciable formation of phase structures in the polymer solutions. In the present paper, the consequence of this facile crosslinking to the phase structures and the 
mechanical properties of the heterogeneous network polymers will be described.

\section{EXPERIMENTAL}

Poly(L-glutamic acid) $(\mathrm{PGA})\left(M_{w}=4.5 \times 10^{4}\right)$ was prepared by saponifying poly $(\gamma$-methly L-glutamate) purchased from Ajinomoto Co. Ltd. ${ }^{13}$ Poly(oxyethylene glycol)s (PEG), supplied by Sanyo Chemical Industries, had number-average molecular weights of 330,570 , and 880 , based on acetylation; these are designated as PEG300, PEG600, and PEG900, respectively. Polyether diisocyanates (PEGDI) were synthesized by allowing the dried PEG to react with twice the number of moles of hexamethlyene diisocyanate at $80^{\circ} \mathrm{C}$ under a nitrogen stream for $5-6 \mathrm{~h}$ until half the NCO groups were consumed.

PEGDI-based heterogeneous network polymers containing varying proportions of PGA $(30,40,50$, 60 , and $70 \%$ by weight) were synthesized. For example, $1.25 \mathrm{~g}$ PGA was dissolved in $30 \mathrm{~m} l$ anhydrous $N, N$-dimetylacetamide (DMA) by gentle heating; $1.25 \mathrm{~g}$ PEGDI derived from PEG300 was added; the homogeneous reaction mixture was poured into a teflon-coated glass dish without a lid, and allowed to stand at $70^{\circ} \mathrm{C}$ in a thermostatted oven for $c a .24 \mathrm{~h}$ while the DMA was allowed to evaporate gradually. This particular polymer is abbreviated as PGA-PEG300DI(50/50); the weight ratio of the two components is indicated in the parentheses. The preparation of PGA-PEG has been given elsewhere. $^{6}$

The instruments and the conditions for dynamic mechanical measurements, infrared spectroscopy, $\mathrm{X}$-ray diffractions and polarizing optical microscopy were comparable to those given previously. ${ }^{8}$ Stress-strain measurements were performed on a Shimazu Autograph IM-100 with an elongation speed of $100 \mathrm{~mm} / \mathrm{min}$ at $25^{\circ} \mathrm{C}$; the specimens were strips, $0.6 \times 0.8 \times 40 \mathrm{~mm}$, cut out from the polymer sheets.

\section{RESULTS AND DISCUSSION}

To prevent evolved carbon dioxide bubbles from being trapped in the final products, the crosslinking of PGA with PEGDI must be completed before the system becomes too viscous. Fortunately, this was succesfully done by working with dilute casting soultions, in which the initial concentrations of the

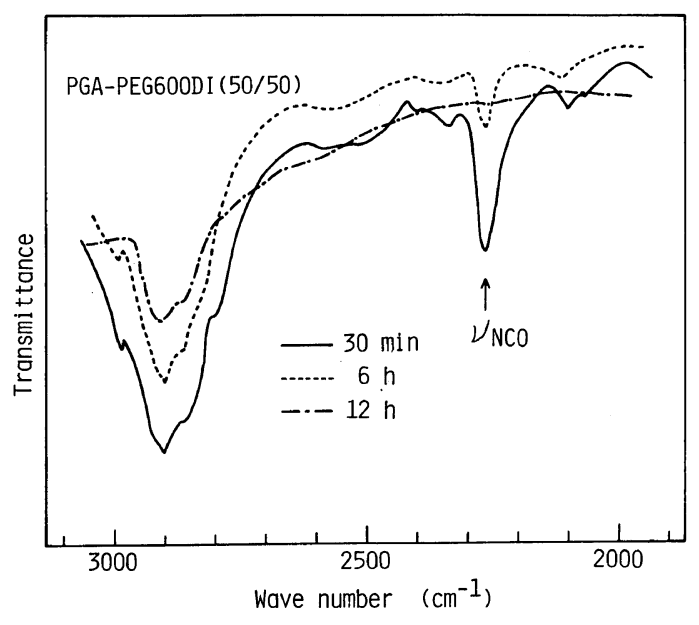

Figure 1. Dissipation of NCO groups of crosslinking agent during the preparation of PGA-PEG600DI(50/50).

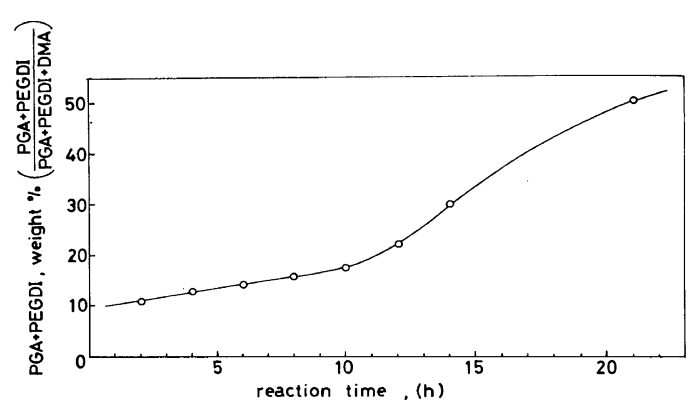

Figure 2. Change in polymer concentration during the prearation of PGA-PEG600DI(50/50).

component polymers did not exceed 10 percent by weight. As monitored by the decrease in the infrared absorption at $2,270 \mathrm{~cm}^{-1}$, all the isocyanato groups in PEGDI disappeared in $12-14 \mathrm{~h}$ at $70^{\circ} \mathrm{C}$; in the meantime the slow evaporation of the solvent, DMA, caused an increase in the polymer concentration only up to 20 percent, as illustrated in Figures 1 and 2. As the DMA further evaporated from these extensively swollen crosslinked polymers to dryness, transparent solid films were obtained and some of their properties are given in Table $\mathbf{I}$.

The infrared spectra of the solid films had intense absorptions at 610 (amide V) and $410 \mathrm{~cm}^{-1}$ indicating the $\alpha$-helix to be the predominant conformation of the PGA, although minor peaks at 700 and $650 \mathrm{~cm}^{-1}$ suggested that the presence of the $\beta$ - and the random coil conformations could not completely be ruled out. As shown in Figure 3, the polarizing 


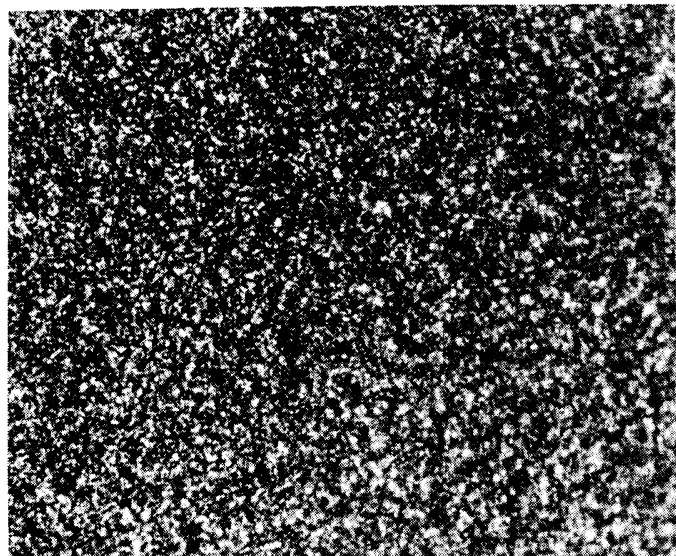

(a)

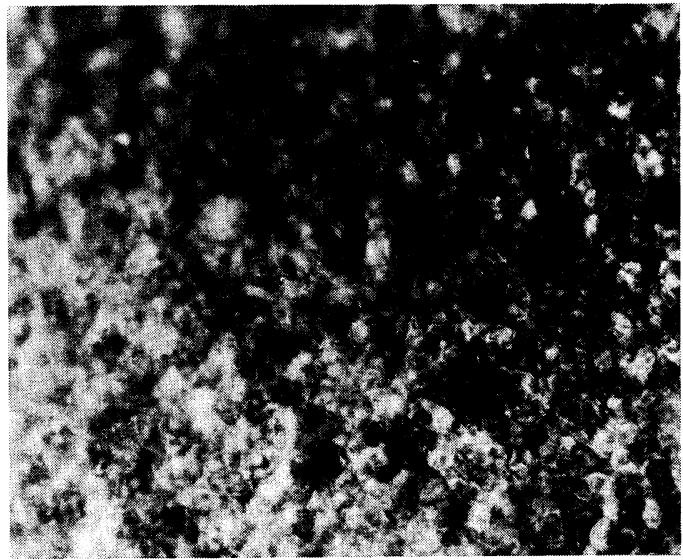

(d)

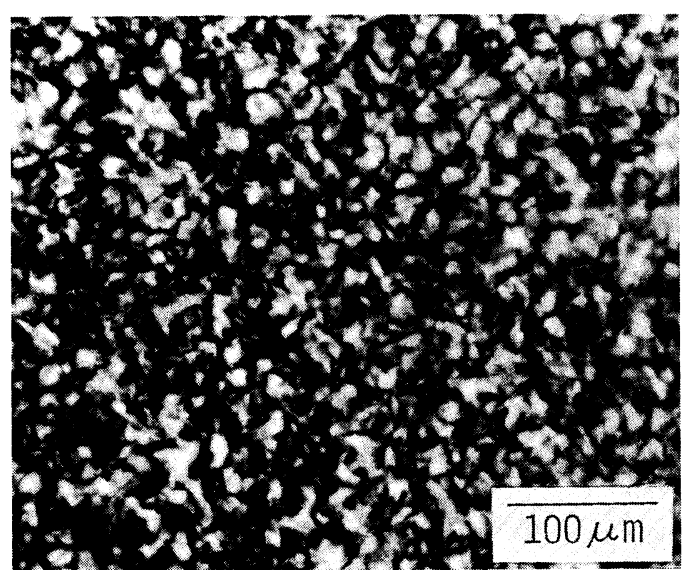

(b)

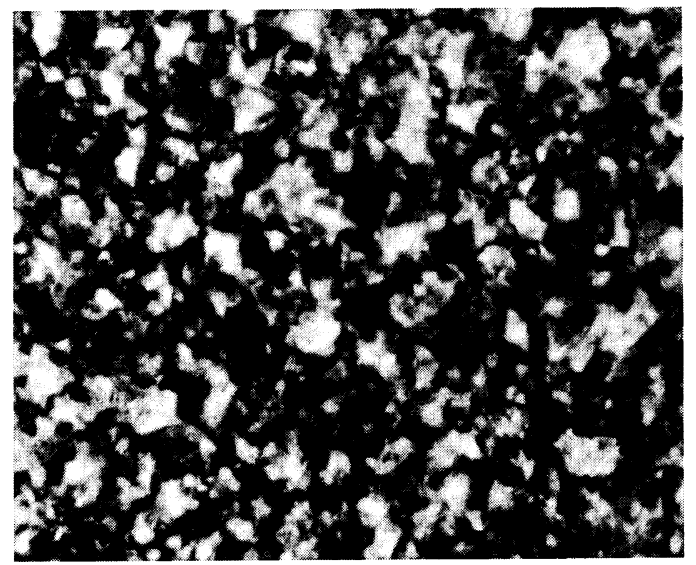

(c)

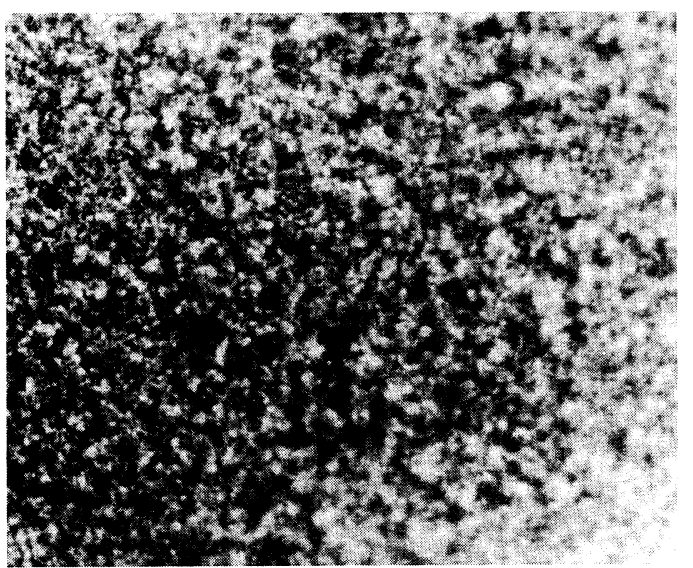

(e)

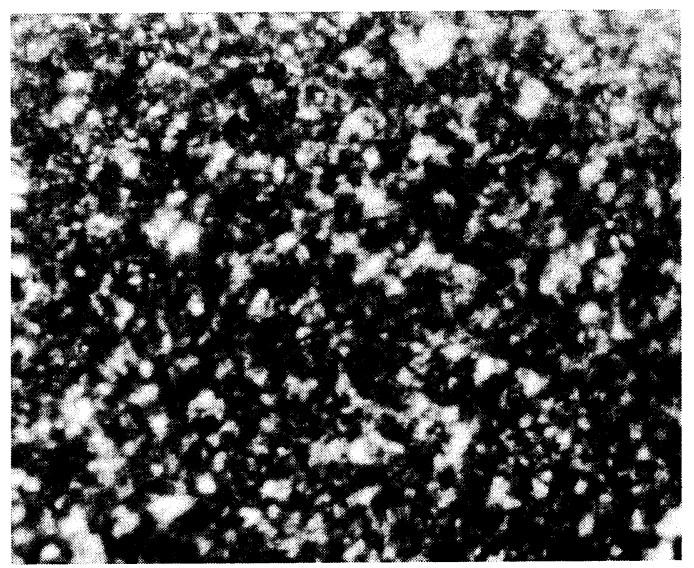

(f)

Figure 3. Polarizing optical micrographs of heterogeneous network polymers: (a) PGA-PEG300(50/50); (b) PGA-PEG300(60/40); (c) PGA-PEG300(70/30); (d) PGA-PEG300DI(50/50); (e) PGAPEG300DI(60/40); (f) PGA-PEG300DI(70/30). 
T. TAnaka, T. Mori, K. Ogawa, and R. TAnaKa

Table I. Characterization of network polymers

\begin{tabular}{|c|c|c|c|c|c|c|c|}
\hline \multirow{2}{*}{ Sample } & \multirow{2}{*}{$K^{\mathrm{a}}$} & \multirow{2}{*}{$\mathrm{Gel} / \% \mathbf{o}^{\mathrm{b}}$} & $v^{\mathfrak{c}} \times 10^{3}$ & \multirow{2}{*}{$q^{\mathrm{d}}$} & \multirow{2}{*}{$\frac{\rho^{\mathrm{e}}}{\mathrm{g} \mathrm{cm}^{-3}}$} & \multirow{2}{*}{$\frac{C^{\mathrm{f}} \times 10^{3}}{\mathrm{~mol} \mathrm{cm^{-3 }}}$} & \multirow{2}{*}{$\frac{T^{\mathrm{g}} \tan \delta_{\max }}{{ }^{\circ} \mathrm{C}}$} \\
\hline & & & $\mathrm{mol} \mathrm{cm}^{-3}$ & & & & \\
\hline \multicolumn{8}{|c|}{ PGA-PEG300DI } \\
\hline$(30 / 70)$ & 1.1 & 91.5 & 2.73 & 2.95 & 1.241 & 0.15 & 45 \\
\hline$(40 / 60)$ & 1.6 & 92.6 & 2.38 & 3.16 & 1.263 & 1.53 & 74 \\
\hline$(50 / 50)$ & 2.5 & 92.6 & 2.02 & 3.41 & 1.284 & 2.96 & 90 \\
\hline$(60 / 40)$ & 3.7 & 97.5 & 1.64 & 3.17 & 1.305 & 4.43 & 113 \\
\hline$(70 / 30)$ & 5.8 & 98.3 & 1.24 & 3.52 & 1.311 & 5.88 & 133 \\
\hline \multicolumn{8}{|c|}{ PGA-PEG600DI } \\
\hline$(30 / 70)$ & 1.6 & 87.5 & 1.84 & 3.22 & 1.232 & 1.02 & 30 \\
\hline$(40 / 60)$ & 2.4 & 93.2 & 1.61 & 3.24 & 1.255 & 2.28 & 47 \\
\hline$(50 / 50)$ & 3.6 & 95.9 & 1.37 & 3.37 & 1.280 & 3.59 & 76 \\
\hline$(60 / 40)$ & 5.4 & 95.9 & 1.12 & 3.48 & 1.305 & 4.95 & 99 \\
\hline$(70 / 30)$ & 8.5 & 99.2 & 0.85 & 3.66 & 1.319 & 6.31 & 140 \\
\hline \multicolumn{8}{|c|}{ PGA-PEG900DI } \\
\hline$(30 / 70)$ & 2.1 & 82.7 & 1.39 & 3.92 & 1.227 & 1.46 & 13 \\
\hline$(40 / 60)$ & 3.2 & 86.7 & 1.21 & 4.15 & 1.249 & 2.66 & 33 \\
\hline$(50 / 50)$ & 4.8 & 95.2 & 1.03 & 4.41 & 1.277 & 3.92 & 57 \\
\hline$(60 / 40)$ & 7.2 & 98.0 & 0.84 & 4.02 & 1.300 & 5.21 & 95 \\
\hline$(70 / 30)$ & 11.2 & 98.3 & 0.64 & - & 1.320 & 6.52 & - \\
\hline
\end{tabular}

a $K=\left[\mathrm{CO}_{2} \mathrm{H}\right] /[\mathrm{NCO}]$ in the original reaction mixtures.

b Extracted with $N, N$-dimethylacetamide.

c Crosslink density based on $K$.

d Swell ratio in $\mathrm{N}, \mathrm{N}$-dimethylacetamide.

e Density at $25^{\circ} \mathrm{C}$ measured by flotation method in cyclohexane $-\mathrm{CCl}_{4}$.

f Concentration of free carboxyl groups.

g Measured with Rheovibron DDV IIC at $110 \mathrm{~Hz}$.

optical micrographs of PGA-PEGDIs, at first glance, resemble those of PGA-PEGs. But in PGA-PEGDIs, the proportion of the dark regions was larger and the bright spots were much more dispersed and blurred than in PGA-PEGs. This indicates that the amount and the orderlyness of the optically anisotropic domains are meagrer in PGA-PEGDI systems. This was also borne out by $\mathrm{X}$-ray diffraction patterns in that the reflection $\left(2 \theta=6-9^{\circ}\right)$, presumably due to the $(10 \overline{1} 0)$ planes of hexagonally packed $\alpha$-helices of PGA, was much weaker in PGA-PEGDIs than in PGA-PEGs.

A mixture of PGA and PEG is known to form lyotropic cholesteric liquid crystals in solvents such as DMA, dimethylformamide, or dioxane-water, and the polymer concentration of the 'A' point, at which the liquid crystalline spherulites begin to phase-separate, is $c a .12-15$ percent by weight at $30^{\circ} \mathrm{C}{ }^{12,14}$ Because this concentration should be higher at $70^{\circ} \mathrm{C}$, the reaction temperature, the crosslinking reaction intervened, therefore, in the
PGA-PEGDI systems before the 'A' point could be reached. The preactically isotropic, highly swollen, crosslinked polymers may have coagulated and some local structures may have been formed while the solvent was evaporating further; nevertheless, the extensive formation of the cholesteric liquid crystalline organizations, which were prevalent in PGA-PEG, and the phase separation may have, by and large, been prevented by the crosslinks constructed in advance.

The dynamic mechanical properties of PGAPEG300DI and PGA-PEG600DI are shown in Figures 4 and 5 . The position of the $\tan \delta$ maximum shifted to a higher temperature, and the magnitude of $E^{\prime}$ above the transition temperature increased as the PGA content was raised (with concomitant decrease in the crosslink density, which would have caused a reverse trend); i.e., the copolymerization effect predominated over the effect of crosslink density. Whereas in PGA-PEG, the shape and the magnitude of $\tan \delta$ curves changed quite abruptly 


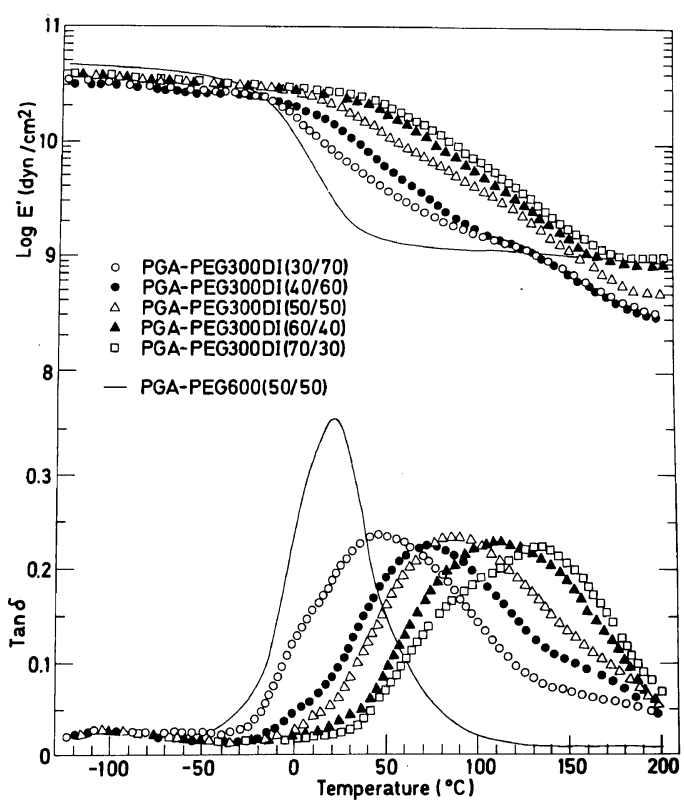

Figure 4. Dynamic mechanical properties of PGAPEG300DI at $110 \mathrm{~Hz}$.

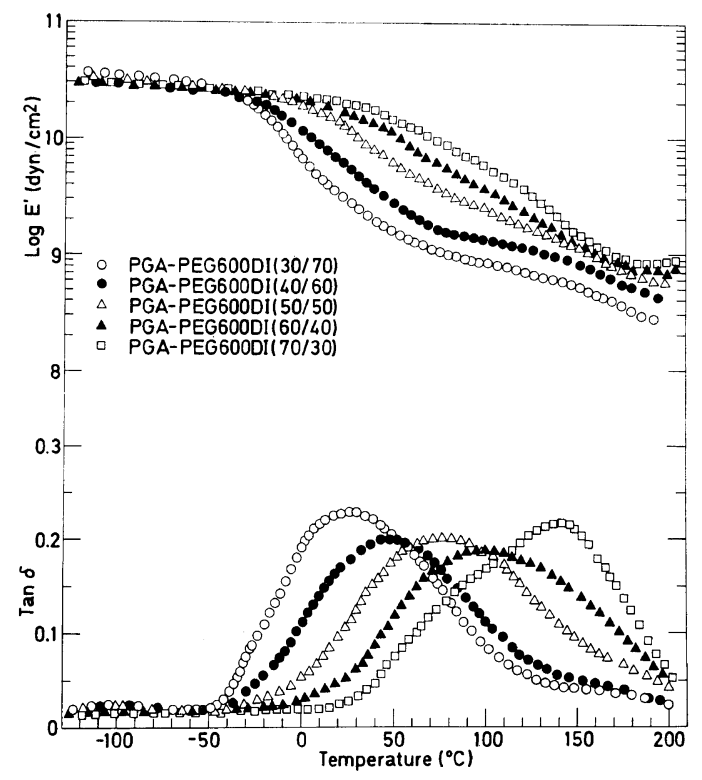

Figure 5. Dynamic mechanical properties of PGAPEG600DI at $110 \mathrm{~Hz}$.

near a PGA content of 50 percent, and an occurrence of phase inversion was demonstrated; ${ }^{8}$ the values of $\tan \delta$ maxima for the PGA-PEGDI systems remained nearly constant regardless of the PGA content, and no discontinuous change in phase structure was recognized. To account for this and the polarizing microscopic as well as the $\mathrm{X}$-ray observations, the main relaxational peak has to be attributed to the micro-Brownian motions of the amorphous regions that consist of well-mixed PGA and PEGDI segments. The existence of such amorphous regions as continuous phases is consistent with the view that the isotropic casting solutions were cured, so to speek, and that their 'structure-lessness' was somehow incorporated into the solid films.

The stress-strain curves of PGA-PEG600DI at $25 \mathrm{C}$ are shown in Figure 6 . Compared with the behavior of the corresponding PGA-PEG600 (shown in broken lines), PGA-PEG600DI turned out to be both stronger and tougher. The high elastic moduli and the large elongations at break were efficiently provided by PGA and PEGDI, respectively, because the extent of crosslinking was very high and much of the stress was carried by the wellmixed PGA-PEGDI continuous phase.One might still argue that the introduction of a urethane linkage and six methylene groups at both ends of PEG should simply give another series of crosslinking

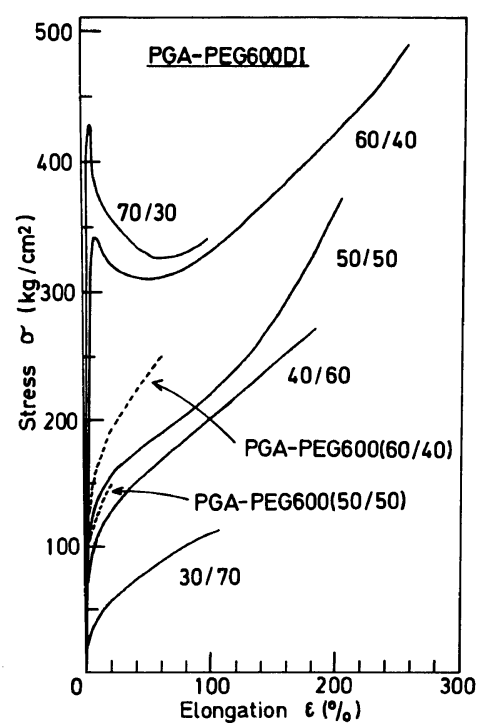

Figure 6. Stress-strain curves of PGA-PEG600DI at $25^{\circ} \mathrm{C}$. Each curve is a representative of at least five similar measurements that were reproducible within $\mathrm{ca} .10 \%$ in both strength and elongation. 
agents completely different from PEG, and that the difference in mechanical properties between PGA-PEG and PGA-PEGDI should be accounted for within this context. Nevertheless, the effect of good mixing in the continuous phase of PGA-PEGDI induced by the 'early' crosslinking reaction cannot be discredited, since the presence of the less polar hexamethylene groups could have made the two components (PGA and PEG tend to complex each other and are compatible within a range of compositions) ${ }^{12}$ less compatible with each other, thus causing more phase separation than PGA-PEG if crosslinking had occurred at a much later stage in the solvent evaporation.

\section{CONCLUSIONS}

Poly(L-glutamic acid) can be readily crosslinked with polyether diisocyanates in dimethylacetamide. Because the croosslinking precedes any structureforming processes in the casting solutions, a very efficient admixture of the two component polymers is accomplished in the stress-carrying amorphous phase in the solid films so that strong and tough composite materials can be obtained.

Acknowledgment. The authors are grateful to the support by the Grant-in-Aid for Developmental Scientific Research (No. 285208) from the Ministry of Education, Japan.

\section{REFERENCES}

1. A. Donatelli, L. H. Sperling, and D. A. Thomas, J. Appl. Polym. Sci., 21, 1189 (1977).

2. K. C. Frish, D. Klempner, S. Migdal, and A. P. Dunlop, J. Appl. Polym. Sci., 19, 1893 (1975).

3. G. Allen, M. J. Bowden, D. J. Blundall, G. M. Jaffé, J. Vyvoda, and T. White, Polymer, 14, 604 (1973).

4. K. Shibayama and Y. Suzuki, Kobunshi Kagaku, 23, 24 (1966).

5. C. H. Bamford, G. E. Eastmond, and D. Whittle, Polymer, 16, 377 (1975).

6. T. Mori, Y. Kuchihara, R. Tanaka, and T. Tanaka, $J$. Polym. Sci., Polym. Phys. Ed., 12, 501 (1974).

7. T. Mori, R. Tanaka, and T. Tanaka, J. Polym. Sci., Polym. Phys. Ed., 13, 1633 (1975).

8. T. Mori, K. Ogawa, and T. Tanaka, J. Appl. Polym. Sci., 21, 3381 (1977).

9. T. Mori, R. Tanaka, and T. Tanaka, J. Appl. Polym. Sci., 22, 2817 (1978).

10. T. Mori, T. Yamada, R. Tanaka, and T. Tanaka, Kobunshi Ronbunshu, 35, 323 (1978).

11. T. Mori, T. Yamada, R. Tanaka, and T. Tanaka, unpublished result.

12. T. Tanaka, T. Mori, T. Tsutsui, S. Ohno, and R. Tanaka, J. Macromol. Sci. Phys., in press.

13. Y. Takeda, K. Okuda, and S. Sakurai (Ajinomoto Co. Ltd.), Japanese Patent 3290 (1965); Chem. Abstr., 61, P10, 822c (1964).

14. T. Mori and R. Tanaka, unpublished result; see also T. Tsutsui and T. Tanaka, Chem. Lett., 1315 (1976). 